\title{
On Polar Questions
}

\author{
Robert van Rooy and Marie Šafářová \\ ILLC, University of Amsterdam
}

\section{Introduction}

According to the popular Hamblin/Groenendijk \& Stokhof (Hamblin 1973, Groenendijk \& Stokhof 1984) semantic theories of questions, the meaning of a question $\mathrm{Q}$ is a set of propositions that are "good" answers to the question. Thus the meaning of the question Is Luke right? is a set containing the proposition Luke is right and its complement, Luke is not right. The same holds for the negative polar question Is Luke not right?

$$
\begin{aligned}
& {[[\text { Is Luke right? }]]=[[\text { Is Luke not right? }]]=} \\
& \{\lambda w[\text { Luke is right in } w], \lambda w[\text { Luke is not right in } w]\}
\end{aligned}
$$

The meanings of alternative questions (alQs) like Is Luke right or is Mark right? can be seen as special cases of wh-questions. For example, in Hamblin's system (with von Stechow's extension regarding the meaning of the disjunctive connective) it is the union of two sets of propositions: those containing the proposition that Luke is right and those containing the proposition Mark is right.

$[[$ Is Luke right or is Mark right? $]]=[[$ Luke is right $]] \cup[[$ Mark is right $]]=$ $\{\lambda w[$ Luke is right in $w]\} \cup\{\lambda w[$ Mark is right in $w]\}=$ $\{\lambda w[$ Luke is right in $w], \lambda w[$ Mark is right in $w]\}$

Consequently, the semantics of an alternative question composed of two polar alternatives, like Is Luke right or is Luke not right? is the same as the semantics of the polar question in (1).

[[Is Luke right or is Luke not right?]]= $\{\lambda w[$ Luke is right in $w], \lambda w[$ Luke is not right in $w]\}$

According to Hamblin's theory, there is thus no difference between positive polar questions (PPQs), negative polar questions (NPQs), and alternative questions (composed of two polar alternatives) in terms of their meaning. Groenendijk \& Stokhof (1984) - making use of type-shift - basically predict the same. There is evidence, however, that the three types of questions are not always interchangeable. In the next section, we will give examples of contexts where the different polar questions and the alternative questions either cannot occur in free variation at all, or they receive different readings. In section 3 of this paper, we will summarize possible semantic and pragmatic approaches to the data and point out why they are not satisfactory. In section 4 and 5, we will offer a decision-theoretic account and show in detail how it can solve the previously discussed puzzles. The final section summarizes the proposal. 


\section{Differences between Positive, Negative, and Alternative Questions}

\subsection{Positive versus Negative Polar Questions}

Although the positive and negative polar question Is Luke right? and Is Luke not right? are alike in that both the yes and the no answer are the (only) complete answers to both questions, they cannot always be used interchangeably without change of meaning. Quite convincing examples are rhetorical questions, or tag questions like (4):

a. John is nice, isn't he?

b. John isn't such a bad guy, is he?

The tag question (4a) is used if the speaker wants to indicate that John is nice, while in (4b) she wants to indicate that he isn't a bad guy.

The implicature associated with the negative tag question in (4a) might suggest that a negative polar question always indicates that the speaker takes the positive proposition to be more likely. As it turns out, however, negative polar questions can also be used if the speaker expects a negative answer. Consider Ladd's (1981) original example where the relevant polar question is highlighted. In this case, Kathleen uses the negative polar question and expects a positive answer.

Situation: Kathleen and Jeff have just come from Chicago on the Greyhound bus to visit Bob in Ithaca.

a. Bob: You guys must be starving. You want to get something to eat?

b. Kathleen: Yeah, isn't there a vegetarian restaurant around here?

- Moosewood, or something like that?

c. Bob: Gee, you've heard about Moosewood all the way out in Chicago huh? OK, let's go there.

In the following example, however, she rather expects the negative answer.

Situation: Bob is visiting Katleen and Jeff in Chicago while attending the CLS.

a. Bob: I'd like to take you guys out to dinner while I'm here - we'd

- have time to go somewhere around here before the evening session tonight, don't you think?

b. Kathleen: I guess, but there's not really any place to go to in Hyde Park.

c. Bob: Oh, really, isn't there a vegetarian restaurant around here?

d. Kathleen: No, about all we can get is hamburgers and souvlaki.

Ladd (1981) argues that to account for this distinction, we have to assume a genuine syntactic/semantic ambiguity here, involving a difference in scope of the negation. We will come back to these examples and see in how far we have to assume that the difference between (5b) and (6c) involves a different use of negation. 
Another type of contexts in which negative polar questions are not quite interchangeable with positive polar questions are those where knowledge that the negative proposition is true gets the speaker closer to her conversation goal. One example is that of a medical examination, as observed on hospital fliers:Is your child apathetic? Is your child not eating properly? Has (s)he not been sleeping for the last three days? Similarly, one can test the ecological behavior of fellow citizens by asking them Do you turn off appliances when they are not in use? Do you not dump waste? Do you not run the water while brushing your teeth?

\subsection{Alternative versus Polar Questions}

Bolinger (1978) noted that polar questions and alternative questions cannot always be considered variants of each other. ${ }^{1}$ Based on Bolinger's observations and our own collection of data, we grouped contexts in which this is the case into the following categories:

\section{Requests}

While polar questions are common as pleas or requests, it seems strange to use an alternative question in the same context. Compare (7a) to (7b), or (8a) to (8b).
a. Will you marry me?
b. Will you marry me or not?
(train conductor to a passenger)
a. May I see your ticket?
b. May I see your ticket or not?

\section{Drawing Inferences}

When drawing inferences, either linguistic (as in (9)), or situational (as in (10), when somebody just came into the room with her jacket soaking wet), polar questions appear to be more appropriate than alternative questions.
a. A: I just saw David.
B: Is David back from Toronto?
b. B: Is David back from Toronto or not?
a. Is it raining?
b. Is it raining or not?

\section{Invitations}

Invitations are of ten impolite (or impossible, as in the elided (12b)) if uttered as alternative questions. In some situations, though, it may be preferable to use an alternative question like (11b) rather than the polar one in (11a), e.g., if the speaker does not want to come across as being pushy. 
a. Do you want something to drink?

b. Do you want something to drink or not?
a. Cigarette?
b. Cigarette or not?

\section{Conversation Starters}

If the goal of conversation participants is mainly to communicate friendly intentions towards each other and to engage in a polite exchange for a certain period of time, the use of the polar question in (13a) is more likely than the alternative question in (13b).
a. Do you like to play golf?
b. Do you like to play golf or not?

\section{Rhetorical Questions}

It seems impossible to realize rhetorical questions like (14) as alternative questions. Although with rhetorical questions, an answer is not expected, we assume that their use is parasitical on the proper use of questions and the contrast between (14a) and (14b) should thus be accounted for.
a. Are you crazy?
b. Are you crazy or not?

It seems that at least with one type of negative polar questions, alternative question variants are not possible. Thus (15b) is not a paraphrase of (15a); it is actually difficult to think of a context in which it could be used (though (15c) is acceptable, so this may simply be due to a convention on the order of alternatives in the question).

a. Don't you have to ask your parents first?

b. Don't you have to ask your parents first or do you?

c. Do you have to ask your parents first or don't you?

\section{Towards a pragmatic account}

Why can't positive polar questions, negative polar questions, and alternative questions always be considered variants of each other? The first possible explanation is that this is due to the fact that these questions have a different semantics. Thus, contrary to what is standardly assumed, one might say that (1) and (3) do not have the same denotation. Perhaps the most obvious way to account for this would be to say that the positive and negative PQ in (1) just denote the singleton set containing the proposition that Luke is right, $\{q\}$, and that he is not right, $\{\neg q\}$, respectively, while (3) denotes the set that contains both, $\{q, \neg q\}$. In fact, extending Hamblin's (1973) 
semantics for $w h$-questions to yes/no-questions immediately gives rise to this effect. According to Hamblin's semantics the meaning of a wh-question like Who came? is the following set of propositions: $\{\lambda w[\mathrm{~d}$ came in $w]: d \in D\}$. When we extend this analysis to $n$-ary wh-questions, the following results (where $\vec{x}(\vec{d})$ is an $n$-ary sequence of variables (individuals)):

$$
[[? \vec{x} P \vec{x}]] \quad-\quad\left\{\lambda w\left|\vec{d} \in I_{u}(P)\right|: \ddot{d} \in I^{n}\right\}
$$

Let us call this denotation the extended Hamblin denotation. Polar questions result when $n=0$, and the predicted denotation of ?q then is $\{q\}$.

Both Hamblin and Groenendijk \& Stokhof assume that the meaning of a yes-no question is its set of complete answers. On this reading, the above suggestion by itself is not enough to account for our data - it seems necessary to 'reinterpret' the question-meaning. As a matter of fact, Roberts (1996) makes use of the above discussed extended Hamblin denotation of questions and proposes that polar questions give rise to the denotation as suggested above. However, she does not consider the elements of a question-denotation to be the possible answers to the question, but rather the propositions in the truth value of which the questioner is interested. Although this interpretation is appealing, the proposal is not enough to solve our problem: assuming a two-valued semantics, if somebody is interested in learning if $q$ is the case, she should be equally interested in learning if $\neg q$ is the case. The proposal thus does not make a distinction between the positive and negative proposition that can account for the difference between PPQs, NPQs, and AlQs.

The above discussion suggests that to make a distinction between the positive and the negative proposition, we should look beyond the semantic factors, namely to pragmatics. A possible pragmatic way to make the needed distinction was suggested by H. Zeevat (p.c.) who proposed a solution in terms of 'weak presupposition'. A weak presupposition is weaker than a real presupposition in that it can be triggered by embedded clauses (as in (16) for the particle too), or by a linguistic or a behavioral suggestion in the context.

A: John dreamt that Mary was in Spain.

B: Harry is in Spain, too.

Weak presupposition is a kind of minimal evidence in the common ground. This allows for a context to contain a weak presupposition both for a positive proposition, as well as for its negation. The difference between different types of questions would then be captured by differences in the weak presuppositions as follows: 


\begin{tabular}{|c|c|c|}
\hline TYPE OF QUESTION & ABBREVIATION & CONTEXT \\
\hline Positive polar question & $? q$ & $\begin{array}{c}\text { no weak } \\
\text { presupposition } \\
\text { or } \\
\text { weak presupposition } \\
\text { for } q\end{array}$ \\
\hline Negative polar question & $? \neg q$ & $\begin{array}{c}\text { weak presupposition } \\
\text { for } \neg q\end{array}$ \\
\hline Alternative question & $? q$ or $\neg q$ & $\begin{array}{c}\text { weak } \\
\text { presupposition for } q \\
\text { and } \neg q\end{array}$ \\
\hline
\end{tabular}

As a matter of fact, Büring and Gunlogson (2000) offer somewhat comparable intuitions in terms of their 'Evidence Conditions':

- PPQ: there is no compelling evidence against $q$

- ONPQ: there is no compelling evidence for $q$

- INPQ: there is compelling evidence against $q$

where $\mathrm{q}$ is the proposition expressed by the question, ONPQ stands for Ladd's 'outer-negation polar question' and INPQ for 'inner-negation polar question'. (We will return to this distinction below.)

One argument against the weak-presupposition analysis is based on the fact that a negative polar question like ? $\neg q$ can be used in contexts which contain no evidence for either $q$ or $\neg q$. For example, when browsing the internet, a user may come across web sites asking Do you not have any friends? Then click on this button... or Have you not been able to receive credit from your financial institution to back up your business activities? Then.... Given that the creators of the web sites have no prior information about the user's private life or financial situation, they could not possibly pose their questions based on prior evidence. Rather, they use negative polar questions instead of the positive ones because it is the truth of the negative proposition that is relevant for the user's intended next step (click on a button).

Another example of an unbiased context is that of medical examination, already mentioned above. The choice of questions (positive or negative) is determined by the list of symptoms for a given illness. The doctor would most likely not ask Has she not been apathetic? or Is she smiling or playful?, because if it were so, it would not help her to reach a diagnosis. The goal of the doctor-patient discourse is not to determine how well we perform in certain areas, but where our performance is below average. In other words, it seems that whether the speaker opts for a negative or for a positive polar question at least partly depends on her conversation goals. This point is not accounted for by either of the approaches summarized above.

Another relevant example is that of a context biased with respect to a proposition in which an alternative question can be posed. Thus in (17), B can easily 
respond with an alternative question to A's utterance if she thinks that $\mathrm{A}$ is in a position to answer the question reliably, even though there is a weak presupposition in the context for Bill killed the neighbor's dog. Similarly, in (18), the speaker can use both a positive polar question or an alternative question in a context biased for a proposition (if we assume that bias can also be generated by inferences).

a. A: John thinks that Bill killed the neighbor's dog.

b. B: Well, did he kill it or not?

The political situation in the country is unstable.

Is it safe to go there or not?

Finally, there is the 'paradox of positive polar questions': it seems that this type of questions can be used not just in contexts containing positive evidence, but also in contexts with negative evidence. This is in case that a new surprising fact has come up which the speaker is for some reason reluctant to ground, as in (19a) and (20a), or, even more strongly, in (19b) and (20b) with contrastive accents, because she believes the negative proposition to be more likely.

a. Are you telling me to leave?

b. Are YOU telling ME to leave?

(You are not in a position to give me orders.)

a. Is John the boss?

b. Is JOHN the boss?

(I thought Peter was the boss.)

These examples show that the data are more problematic than it would seem at the first blush. We agree with Zeevat that the distinction between the two sorts of questions should be explained in pragmatic terms; moreover, the examples which served to doubt the weak presupposition theory as the general account, also showed that two further factors influence the choice of polar questions over alternative questions (or vice versa): the likelihood of some proposition being true (as in (19) and (20)) and the goals of the speaker/writer-hearer/reader interaction (as in the doctor-patient dialogue). In the next section, we will propose a decision-theoretic description of the question types which incorporates these two features. In general, we will argue that the difference between the various types of polar questions and alternative questions can be captured in terms of the expected utilities of their answers. In this way we don't have to assume that the different types of questions give rise to different denotations.

\section{The utility of answers}

In the semantic/pragmatic analysis of natural language we often take the beliefs of the conversational participants into account. In speech act theory it has always 
been assumed that this is not enough; we should consider their preferences (or desires) as well. Once both beliefs and preferences are taken into account, it becomes natural to make use of decision theory. The decision-theoretic approach to semantics and pragmatics of natural language has been advocated in recent studies (e.g., Merin (1999) and van Rooy (to appear a, b)) to account for, among others, conversational implicatures and the interpretation of questions. In decision theory, an agent's belief-desire state is modelled as a tuple $\langle P, U\rangle$, where $P$ stands for the agent's probability function, which represents her beliefs, and $U$ for her utility function, which together with $P$ models her preferences. Here we will make use of Jeffrey's (1965) decision theory, where utility functions are functions from worlds to real numbers. In this framework, we can determine the expected utility of any proposition $p$ as below.

$$
E U(p)=\sum_{w \in p} P(w) \times U(w)
$$

In particular, we can determine the utility of the tautologous proposition, $T$. This is important, because the expected utility of proposition $T$ measures the value of the current belief-desire state: $E U(\top)=\sum_{w \in \top} P(w) \times U(w)=\sum_{w} P(w) \times U(w)$. From now on we will denote this value by $E U(P, U)$. If $\langle P, U\rangle$ and $\left\langle P^{\prime}, U^{\prime}\right\rangle$ are two different belief-desire states, $E U(P, U)>E U\left(P^{\prime}, U^{\prime}\right)$ means that the agent prefers to have the beliefs and desires as represented in $\langle P, U\rangle$ to the beliefs and desires in $\left\langle P^{\prime}, U^{\prime}\right\rangle$. As we will see below, this value of a belief-desire state will be of crucial importance to determine the utility of new information.

The above noted representation of a belief-desire state is very general; in particular, what the utility function depends on is left open. For our present purposes, we will consider only some specific instantiations. First, let us assume that our agent has a certain goal or desire: the desire to be in a world where $g$ is true, or the goal to make that world actual where $g$ is true. In that case we can assume that the utility function is defined as follows:

$$
U(w)=1 \quad \text { iff } \quad w \in g, 0 \text { otherwise }
$$

Notice that now the value $E U(P, U)$ reduces to the probability of $g, P(g){ }^{2}$

As a second special case, let us assume that our agent just wants to know what the world is like. In that case it is natural to assume that the utility of the worlds crucially depends on the probability function that represents the beliefs of the agent. If the agent's beliefs are represented by probability function $P$, the utility of the world $w$ turns out to be $\log P(w)$ (cf. van Rooy (to appear b)). If we now calculate the value of the state $\langle P, U\rangle$, it turns out that this will equal the negative entropy on the set of all worlds induced by probability function $P$. To show this, we first have to know how to determine the entropy of a partition. Suppose that $Q$ is a set of propositions that partitions the state space. The entropy of $Q$ with respect to $P, E_{P}(Q)$, is then determined as follows: $E_{P}(Q)=-\sum_{q \in Q} P(q) \times \log P(q)$ (cf. Shannon (1948)) . This value measures the uncertainty about which element of $Q$ is true: $E_{P}(Q)$ is maximal in case all elements of $Q$ are equally likely, and $E_{P}(Q)$ 
is 0 - which is the minimal entropy value a partition can receive - if the agent knows for certain which element of $Q$ is the case. If we assume that $W$ denotes the partition corresponding to the set of all worlds, the entropy of $W$ with respect to probability function $P, E_{P}(W)$, is determined as $-\sum_{w} P(w) \times \log P(w)$. Because by assumption $E U(P, U)=\sum_{w} P(w) \times \log P(w)$, this means that $E U(P, U)=$ $-E_{P}(W)$. And this makes sense as well: the entropy of partition $Q$ measures to what extent the elements of $Q$ are equally likely. If an agent wants to know what the world is like, she would prefer to have a probability function that gives rise to a low entropy of partition $W$. And this is what we predict: because $E U(P, U)=$ $-E_{P}(W)$, this value is high if and only if $E_{P}(W)$ is low.

Until now we have only determined the value of the current belief-desire state of an agent. Now we are going to use this to determine how good it is to learn the new information that proposition $q$ is the case. As is standard in decision theory, we will measure the value of this new information in terms of the difference between the values of the information states before and after the agent learned proposition $q$. We will assume that learning proposition $q$ goes by conditionalization: we eliminate all worlds where $q$ is not true and the probabilities among the resulting worlds are distributed as similar as possible as in the prior probability function. We will denote the probability function after conditionalizing $P$ with $q$ by $P_{q}$, and thus assume that $P_{q}(w)=P(w / q)$. We will measure the value of new information $q, U V(q)$, as follows:

$$
U V(q)=E U\left(P_{q}, U\right)-E U(P, U)
$$

What will this utility value come down to in the particular cases we considered above?

First, consider the case where the utility function only depends on the goal proposition $g$. We saw that in this case $E U(P, U)$ reduces to $P(g)$. From this it immediately follows that the value of new information $q$ reduces to the difference between $P(g / q)$ and $P(g)$, i.e. $U V(q)=P(g / q)-P(g)$.

Next, we will consider the situation where our agent just wants to know which world is the actual one. We saw that now the value of a state, $E U(P, U)$, reduces to the negative entropy of the partition $W,-E_{P}(W)$. It immediately follows that the utility value of the new information $q$ reduces to the difference between $-E_{P_{q}}(W)$ and $-E_{P}(W)$, i.e. $U V(q)=-E_{P_{q}}(W)--E_{P}(W)$. This is obviously the same as the reduction of entropy of $W$ due to learning $q, E_{P}(W)-E_{P_{q}}(W)$. In natural circumstances, this, in turn, reduces to the surprisal value of $q$, $\inf (q)$ $=-\log P(q)$, a measure well-known in Information Theory (Shannon (1948)). This surprisal value is defined such that for any two propositions $q$ and $q^{\prime}, \inf (q)>\inf \left(q^{\prime}\right)$ if and only if $P(q)<P\left(q^{\prime}\right)$.

How is this rather technical discussion relevant for our analysis of polar questions? Notice first that in case our agent has a goal $g$ to be(come) true, it holds that $U V(q)>U V(\neg q)$ if and only if $P(g / q)>P(g / \neg q)$ if and only if $P(g / q)>\frac{1}{2}$ (the probability of reaching her goal after finding out that $q$ is higher than after finding out that $\neg q$, i.e., higher than $\frac{1}{2}$ ). Second, in case she wants to know what the world is like it holds that $U V(q)>U V(\neg q)$ if and only if $P(q)<P(\neg q)$. 
In the rest of this paper we will make use of these simple facts to account for our observations discussed above.

\section{Analysis}

Although from a logical point of view there is no essential difference between 'positive' and 'negative' propositions, there exists a remarkable contrast between positive and negative assertions. As discussed in great detail by Givón (1978), negative assertions are much more marked than positive ones. While it is fine to assert $M y$ wife is pregnant in a neutral context, it is odd to claim under those circumstances that My wife isn't pregnant. Givón notes that the negative assertion can only be appropriate in a context in which the positive proposition is taken to be most likely true; only in such a context is the negative statement more informative than its positive counterpart. For an arbitrary sentence $q$ asserting that a certain event took/takes place or that a certain state occurs, the sentence most likely expresses a falsehood if used out of context. Thus, by default, negative statements are less informative than positive ones, which explains why positive statements are the rule and their negative counterparts the exception. Assuming that we choose expressions that make informative statements, Givón argues that negative statements can be used appropriately only in case this default assumption is overruled, thereby explaining the triggered implicature/presupposition of negative statements.

Where Givón seeks to explain the difference in distribution between positive and negative statements, we want to explain the difference in distribution between positive and negative polar questions. In fact, our explanation is closely connected with Givón's proposal: we want to argue that in many circumstances, whether a positive or negative polar question is asked depends on the informativity of the positive and negative statements/answers (see also Han (1998)). Some of the examples discussed in section 2, however, suggest that informativity by itself won't be enough: the goal of asking a question is not always (just) to receive information. Therefore, we will propose that the speaker prefers to ask that type of polar question for which the utility of the positive answer is higher or equal to that of its negative counterpart:

$$
U V(q)>U V(\neg q)
$$

We will consider two most common cases which can increase the utility of the positive answer over the negative one, namely

1. after learning the proposition $q$ it is more likely that one reaches a desirable $g$-world than when its negation is true, i.e., $P(g / q)>P(g / \neg q)$, or

2. the information value of the proposition $q$ being higher than the information value of its negation and thus $U V(q)>U V(\neg q)$ if $U V(q)=\inf (q)=$ $\log \frac{1}{P(q)}$.

The first case is most obviously instantiated in polar questions used as pleas or requests; the goal of the questioner coincides with the proposition expressed by the 
positive answer. The second case is exemplified by questions used as requests for information. For negative polar question, we assume that the utility value of the negative proposition is higher than that of the positive one, i.e. $U V(\neg q)>U V(q)$. It follows that bipolar questions are preferred in case both propositions are (equally) optimal, and thus $U V(q) \approx U V(\neg q)$.

In the following sections we will give a somewhat more detailed analysis of the examples discussed in section 2.

\subsection{Positive Polar Questions}

$$
U V(q)>U V(\neg q)
$$

Many examples of (positive) polar questions seem to occur in contexts where the speaker has a goal and prefers worlds where she has reached that goal to others. On the simplifying assumption that we have one goal - the proposition $g$, we have seen that in these cases $U V(q)>U V(\neg q)$ if and only if $P(g / q)>\frac{1}{2}$. As noted above, this is most obvious with questions-invitations, pleas, or with questions of the sort Do you know how to repair a punctured tire? or Can you help me carry these boxes? The goal $g$ of asking question ? $q$ is for $q$ to become true: $g=q$. The same seems to be going on for invitations. Notice that when $g=q$, the utility of the positive answer will by necessity be higher than the utility of the negative answer.

For pleas and requests it seems natural to assume that the goal equals the questioned proposition. However, the two don't have to be identical for our analysis to be applicable. With conversation starters, for example, we might think of the goal as something like 'having an interesting conversation with someone'. In this case the positive answer is more useful in the sense that it indicates to the conversational participants that they have some common interest which might help to induce an involved conversation. If the hearer answers $\mathrm{No}$, to a conversation starter like $\mathrm{Do}$ you like to play golf?, the speaker has to think of another topic (or give up).

As we saw above, in some cases utility comes down to informativity: $U V(q)>$ $U V(\neg q)$ if and only if $\inf (q)>\inf (\neg q)$. By the definition $\inf (q)=-\log P(q)$, this means that $q$ is now preferred to $\neg q$ in case $P(q)<P(\neg q)$. Given that - as noted by Givón - for most natural language statements $q$ it holds that $P(q)<P(\neg q)$, it follows from our proposal that a yes/no-question is by default stated in terms of a positive polar question. The most obvious examples of positive polar questions where this notion of utility seems to be relevant are falling tag questions such as (21), where the polarity of a tag question contrasts with the polarity of the previous sentence uttered by the same speaker.

$$
\text { John isn't such a bad guy, is he? }
$$

In this case, the speaker takes the declarative sentence to be most likely true, and thus the 'proposition' used by the tag question most likely false, hence, of high informativity.

It is commonly assumed in the literature that for a rhetorical question the negative answer is presupposed to be true. This by itself, however, cannot explain 
the rhetorical effect of a question like (22a). Normally, the point of asking a question is to get some (useful) information. For yes-no questions this means that both answers must be considered to be possible. If only the negative answer is (commonly) presupposed to be true, we get a rhetorical effect. But why do we reach this effect with a (positive) polar question (22a) and not with the alternative question (22b)? The reason is that the agent wants to highlight/suggest that she recently got some indication that the positive proposition might be true after all.

a. Are you crazy?

b. Are you crazy or not?

Questions that check whether some recently received (situational or linguistics) information is really true like Is David back? or Is it raining? can also be explained in terms of utility as informativity. What these examples have in common is the following: the agent has just received some information (linguistic or otherwise) $q$ that was very unexpected (or has an unexpectedly high utility) with respect to her earlier belief-desire state $\langle P, U\rangle$. If she should accept the new information, this would result in a very different belief-desire state $\left\langle P_{q}, U\right\rangle$ : either $P(q)$ is very low, and thus $P_{q}$ would be very different from $P$, or the difference between $E U\left(P_{q}, U\right)$ and $E U(P, U)$ would be unusually large (either positively or negatively). Therefore, the agent double checks by asking questions like in (12a) and (13a). Thus, also in case of grounding questions it is utility as informativity that is crucial. However, here it is with respect to a belief-desire state before the new information is grounded. ${ }^{3}$

Notice that when this last instantiation of utility is at stake, a hearer can conclude from the use of the positive polar question that the questioner had a previous belief or expectation that the negative proposition is true. These implicatures have been analysed recently by Romero \& Han (2001) and they assume - just like we do - that the polar questions are asked to check whether a certain revision of a previous information state should be made. So far, our analyses are similar. ${ }^{4}$ However, their analysis also makes use of another question triggered by the interrogative sentence. They propose that a polar question like WAS John in Hawaii? isn't simply about the fact whether or not John was in Hawaii, but also about how strongly the questioner should believe that John was in Hawaii. If ' $q$ ' stands for 'John was in Hawaii', they propose that WAS John in Hawaii? doesn't simply give rise to partition $\{q, \neg q\}$, but also to $\{P(q)=1, P(q)<1\}$. This latter partition is called the superquestion (cf. Roberts, 1996) generated on the assumption that the questioned proposition gets socalled verum focus. This superquestion is used to derive the epistemic implicature: the answer is still relevant even if the questioner takes $q$ to have a high probability, and thus allows for the answerer to derive the corresponding epistemic implicature.

As we noted already, our analysis of (some instances of) positive polar questions is based on an intuition similar to the proposal employed by Romero \& Han (2001) to explain the epistemic implicatures. The intuition is that the question is asked to check whether one should really revise one's previous information state with the incoming information. However, we simply derive the epistemic implicature from the way a polar question can be useful. ${ }^{5}$ Even with extra assumptions, 
Romero \& Han's explanation still crucially relies on pragmatic (Gricean) reasoning. To our minds, the latter is all one really needs to assume for these cases: information is more useful/relevant if it would trigger a major revision of the previous belief-desire state.

\subsection{Alternative Questions}

$$
U V(q) \approx U V(\neg q)
$$

In many cases, when an agent asks a question, she has certain goals or desires. As we saw above, this is most obviously the case for pleas or requests: the agent prefers certain worlds to others. When asking an alternative question, however, we propose that the speaker is simply in search of the answer to whether $q$ or $\neg q$ holds. She has no bias or wants to give the impression of having no bias with respect to one or the other alternative being either more probable or more useful for her conversation goals. Thus, alternative questions are not good requests or pleas, e.g., in Will you marry me or not? the speaker seems to be manifesting his/her indifference towards the outcome.

As noted by Bolinger, alternative questions can be posed with different degrees of insistence.
a. Did you buy it or not?
b. Did you buy it or didn't you?
c. Did you buy it or didn't you buy it?
d. Did you or did you not buy it?

Note that our proposal does not imply that alternative questions are always neutral with respect to speaker's desires. We take it that in cases like (23), insistence corresponds to a rise in the utility value (with the relation between $U V(q)$ and $U V(\neg q)$ remaining constant). (In fact, the use of the alternative question in general indicates that the search for $q$ or $\neg q$ has a (relatively) high urgency.)

Why are alternative questions sometimes impolite as invitations? Politeness often works via a series of inferences and background assumptions, so it is notoriously difficult to handle theoretically. For that reason, our suggestion is rather tentative: it seems that in the examples in which alternative questions appear to be impolite compared to polar questions (as in (24)), this is due to the implicature that conventionally comes with alternative questions. In particular, the alternative question implies that the utility (and the probability) of the positive answer is equal to the negative answer.

a. Do you want something to drink?

b. Do you want something to drink or not?

If the speaker intends to indicate that the hearer's best interests are on her mind (in decision-theoretic terms, that the value of the hearer's utility is equal to her own), she would choose a question which encodes the hearer's preference. Since with 
invitations, the speaker is presumably offering something that is good or profitable to the hearer, e.g., getting a cup of tea in Can I get you a cup of tea?, she would choose the positive polar question to encode the fact that $U V(q)>U V(\neg q)$. An alternative question would imply that the speaker doesn't care whether the hearer gets a cup of tea or not. This appears to be more polite only in situations where the speaker does not want to appear pushy or insinuating something about the hearer's preferences by implying that $U V(q)=U V(\neg q)$; it can sometimes be more polite to suggest that both the positive and the negative alternative are equally acceptable (as in Do you want to come with us or not?).

\subsection{Negative Polar Questions}

$$
U V(\neg q)>U V(q)
$$

Ladd (1981) distinguishes between inner negation polar questions (INPQs) and outer negation polar questions (ONPQs), an idea which actually goes back to Ladusaw (1979). Ladusaw explains the difference between what Ladd later called INPQs and ONPQs in terms of the different usage of negation. Used descriptively, it negates the proposition contained in the question. Another function of the negation (as in Ladd's ONPQs) is not to negate the proposition contained in the question, but rather to turn the question into a polite suggestion (since the speaker actually believes that the positive proposition is true). According to Büring \& Gunlogson, in languages such as German, an outer negation PQs are marked morphosyntactically. ${ }^{6}$ Thus in (25a), the speaker can think it likely that there actually is no vegetarian restaurant in the neighbourhood, in (25b), this cannot be the case. (25b) would be uttered, for example, in a situation where the speaker recalls that she has been to a vegetarian restaurant in the neighbourhood before.

a. Gibt es kein vegetarisches Restaurant in dieser Gegend?

"Is there no vegetarian restaurant in the neighbourhood?"/

"Isn't there a vegetarian restaurant in the neighbourhood?"

b. Gibt es nicht ein vegetarisches Restaurant in dieser Gegend?

"Isn't there a vegetarian restaurant in the neighbourhood?"

We would like to argue for a different solution; in particular, we propose that Ladd's distinction between inner and outer negation can be discarded. In parallel with positive polar questions, negative polar questions can be divided into two categories:

1. NPQs where $U V(\neg q)>U V(q)$ because the utility function depends solely on the goal proposition $g$ and $P(g / \neg q)>P(g / q)$, and

2. NPQs where $U V(\neg q)>U V(q)$ because the agent only wants to know what the world is like and $\inf (\neg q)>\inf (q)$.

The appropriateness of quiz examples or internet questions like Do you not sort waste? or Does your bank not support your investment? can be explained in a 
straightforward way in terms of the first kind of utility function. ${ }^{7}$ For other examples it is rather informativity that is at issue. Consider first Ladd's ONPQs: (under Büring \& Gunlogson's interpretation) in (25b) the speaker thinks that there is probably a vegetarian restaurant in the neighborhood. In other words, $P(\neg q)<P(q)$ and the surprisal value of $\neg q$ is higher than of the positive proposition, $\inf (\neg q)>$ $\inf (q){ }^{8}$ In the present framework, it is thus not necessary to make any extra assumptions to account for what Ladd calls an ONPQ; the negation used here simply negates the proposition contained in the question.

In a way, it is rather an INPQ like (25a) that is special. We propose that also in such an example the informativity of the negated sentence is higher that that of its positive counterpart. (From our present perspective, Ladd's original terminology is slightly misleading). We agree with Ladd that (25a) with stress on the negation gives rise to a different presupposition/implicature than (25b). However, this fact is not peculiar to negative polar questions, rather, the difference between (25a) and (25b) reflects a difference between uses of questions in general. Note that (25a) on the reading under discussion - functions very much as a grounding question, or a biased question comparable to the example in (26):

Is JOHN the boss?

We often ask this type of questions when we receive some surprising new information that we are reluctant to ground without an additional acknowledgement. ${ }^{9}$ It is thus informativity that is crucial. However - as discussed in section 5.1 - it is not informativity with respect to the current belief-desire state, but rather with respect to a previous one (or better, the state where the last piece of information is not yet grounded). According to Romero \& Han (2001) the questioned proposition in such a situation typically receives verum focus. For negative polar questions this means that the negation must be marked intonationally. Notice, now, that in (25b) the negation cannot be stressed, while in (25a) kein can be. This is most likely the reason why a question like (25a) in which a non-preposed negation occurs can be used to check whether some unexpected information is really true, while a question like (26b) with preposed negation cannot.

\section{Conclusion}

In this paper, we showed that positive polar questions, negative polar questions and alternative questions differ in the conditions of their use. We gave several examples against alternative approaches and on the basis of the discussion we suggested a decision-theoretic solution instead. The difference between polar questions and alternative questions was explained in terms of the utility values of their answers. While for alternative questions, we assumed that the utility value of the positive answer is equal to the utility value of the negative answer, for polar questions this was generally not the case. In particular, for positive polar questions, $U V(q)>$ $U V(\neg q)$, while for negative polar questions, $U V(\neg q)>U V(q)$. 
Furthermore, we have identified two basic types of positive/negative polar questions, namely those where $U V(q)>U V(\neg q)$ because with respect to a goal $g, P(g / q)>P(g / \neg q)$, and those where $U V(q)>U V(\neg q)$ because inf $(\neg q)>$ inf $(q)$. We argued that the distinction between inner negation polar questions and outer negation polar questions is superfluous and that the only problematic case is that of intonationally marked negation. For these examples (parallels of which can be found also in the category of positive polar questions), we followed previous proposals in claiming that the stressed word indicates a 'checking'-function of some new information and thus that informativity has to be calculated with respect to a prior belief-desire state.

\section{Endnotes}

* The authors would like to thank Maria Aloni, Alastair Butler, Paul Dekker, Darrin Hindsill, Katrin Schulz, Henk Zeevat and the audience of the ESSLLI'02 Workshop on Pragmatics in Optimality Theory, the Stuttgart Information Structure workshop and the SALT 13 audience for useful discussions and questions. All remaining mistakes are our own.

1. We can draw a parallel between polar and alternative questions and if and whether, respectively. While if usually does not introduce alternatives, whether is like other wh-words (wh- + either).

2. $E U(P, U)=\sum_{w} P(w) \times U(w)=\sum_{w} P(w) \times(1$, if $w \in g, 0$ else $)=\sum_{w \in g} P(w)=$ $P(g)$.

3. We assume that the same explanation can account for rising tag questions.

4. Disregarding the fact that they consider only informativity, and not utility.

5. Notice that although Romero \& Han's (2001) use of superquestions is motivated by Roberts's (1996) use of superquestions in her analysis of question-strategies, the relation between a question and a superquestion in the two analyses is of a completely different nature. For Roberts, a complete answer to the superquestion induced by an interrogative sentence like Who did JOHN invite?, i.e. Who invited whom?, is also an answer to the question itself. This, however, can obviously not be the case for the superquestion and question induced by an interrogative sentence like WAS John in Hawaii? as used by Romero \& Han: the proposition expressed by Perhaps not resolves Romero \& Han's superquestion, but not the standard yes-no question itself.

6. Actually, they claim that the two types of negative questions are distinguished in this way. However, this claim is probably too strong: according to our informants, with kein unstressed, (25a) below can have both an inner and an outer negation reading.

7. Personal preferences and goals may be distinct. Notice that for these questions the questioner doesn't necessarily prefer a positive answer. Something similar happens if the first author asks the second: Does this suit not make me fat?

8. Romero \& Han (2001) assume that negative polar questions with a so-called 'preposed' negation such as (26b) give rise to the implicature that the speaker takes 
the positive proposition more likely to be true. We extend their proposal to all NPQs, although we respect Romero \& Han's observation that these questions can still be used in two different ways. Our analysis is also in accordance with Ladd's intuition that tag questions are similar tohis ONPQs: we analyze them both in terms of informativity.

9. Although in general, grounding questions can be of both types, i.e., we of ten ask them when the proposition in the question is relevant with respect to achieving our goal.

\section{References}

[Bolinger 1978] Bolinger, D. (1978) 'Yes-no questions are not alternative questions. In H. Hiz (ed.), Questions, 87-105. D. Reidel.

[Büring \& Gunlogson 2000] Büring, D. and C. Gunlogson (2000) 'Aren't positive and negative polar questions the same?' semanticsarchive.net

[Givón 1978] Givón, T. (1978) 'Negation in language: Pragmatics, function, ontology.'In P. Cole (ed.) Syntax and Semantics 9: Pragmatics, 69-112. Academic Press.

[Groenendijk \& Stokhof 1984] Groenendijk, J. and M. Stokhof (1984) Studies on the Semantics of Questions and the Pragmatics of Answers. PhD thesis, University of Amsterdam.

[Hamblin 1973] Hamblin, C. (1973) 'Questions in Montague English', Foundation of Language, 41-53.

[Han 1998] Han, C. (1998) 'Deriving the interpretation of rhetorical questions'. In Proceedings of WCCFL 16, 237-253. CSLI, Stanford.

[Jeffrey 1965] Jeffrey, R.C. (1965) The Logic of Decision. The University of Chicago Press, Chicago.

[Ladd 1981] Ladd, R.D. (1981) 'A first look at the semantics and pragmatics of negative questions and tag questions'. CLS, 17, 164-171. Chicago Linguistic Society.

[Ladusaw 1979] Ladusaw, W. (1979) Polarity Sensitivity as Inherent Scope Relations. PhD thesis, University of Texas.

[Merin 1999] Merin, A. (1999) 'Information, relevance and social decision making'. In J. Ginzburg, L. Moss and M. de Rijke (eds.) Logic, Language and Computation, vol.2. CSLI, Stanford.

[Roberts 1996] Roberts, C. (1996) 'Information structure in discourse'. In J. Hak Yoon and A. Kathol (eds.) Ohio State University Working Papers in Linguistics, vol. 49.

[Romero \& Han 2001] Romero, M. and C. Han (2001) 'On certain epistemic implicatures in yes/no questions'. In R. van Rooy and M. Stokhof (eds.) Proceedings of the 13th Amsterdam Colloquium. ILLC, University of Amsterdam.

[Romero \& Han 2002] Romero, M. and C. Han (2002) 'On negative yes/no questions', submitted to Linguistics and Philosophy.

[Shannon 1948] Shannon, C. (1948) 'The mathematical theory of communication', Bell System Technical Journal, 27, 379-423 and 623-656. 
[van Rooy, to appear (a)] van Rooy, R. (to appear(a)) 'Questioning to resolve decision problems', Linguistics and Philosophy.

[van Rooy, to appear (b)] van Rooy, R. (to appear (b)) 'Utility, informativity, and protocols', Journal of Philosophical Logic. 